bureau: Benecke, Takeda, Tillots, MSD, Cellgene, S. Menegatti: None declared, I. Blijdorp: None declared, H. de Jong: None declared, I. Fluri: None declared, T. Latuhihin: None declared, A. van Kuijk Grant/research support from: UCB, Pfizer, MSD, Janssen, Consultant for: Novartis, Celgene, N. Yeremenko: None declared, D. Baeten Grant/research support from: Pfizer, MSD, AbbVie, UCB, Novartis, Janssen, Boehringer Ingelheim, Consultant for: Pfizer, MSD, AbbVie, UCB, Novartis, Janssen, Boehringer Ingelheim, Eli Lilly, Roche, BMS, Glenmark, This study was funded by an unrestricted grant from Novartis, Employee of: UCB DOI: 10.1136/annrheumdis-2017-eular.1915

\section{AB0115 COMPARISON OF THE BACTERIAL STOOL MICROBIOTA IN ESTABLISHED PSORIATIC ARTHRITIS (PSA) AND PSORIASIS (PSC) - EXPLORATORY ANALYSIS OF PILOT DATA}

M. Castelino ${ }^{1,2}$, M. Tutino ${ }^{2}$, J. Moat ${ }^{3}$, U.Z. Ijaz ${ }^{4}$, R. Parslew ${ }^{5}$, A. Al-Sharqi ${ }^{5}$, R.B. Warren ${ }^{6}$, C. Quince ${ }^{7}$, P. Ho ${ }^{1}$, M. Upton ${ }^{8}$, S. Eyre ${ }^{2}$, A. Barton ${ }^{1,2}{ }^{1}{ }^{1} \mathrm{NIHR}$ Manchester Musculoskeletal Biomedical Research Unit, Central Manchester Foundation Trust, Manchester Academic Health Sciences Centre; ${ }^{2}$ Arthritis Research UK Centre for Genetics and Genomics, Centre for Musculoskeletal Research; ${ }^{3}$ Microbiology and Virology Unit, Institute of Inflammation and Repair, University of Manchester, Manchester; ${ }^{4}$ School of Engineering, University of Glasgow, Glasgow: ${ }^{5}$ Department of Dermatology, Royal Liverpool and Broadgreen Univesrity Hospitals Trust, Liverpool, UK, Liverpool; ${ }^{6}$ The Dermatology Centre, Salford Royal NHS Foundation Trust, University of Manchester, Manchester; ${ }^{7}$ Warwick Medical School, University of Warwick, Warwick; ${ }^{8}$ Faculty of Medicine and Dentistry, University of Plymouth, Plymouth, United Kingdom

Background: Psoriatic arthritis (PsA) is a complex inflammatory condition with both genetic and environmental risk factors contributing to disease. A potential environmental risk factor, known to modify the immune system, is the intestinal microbiota. In PsA there is evidence of intestinal inflammation [1,2] and recently dysbiosis of the gut microbiota has been reported in treatment naïve PsA patients [3]. However, there is no information on the temporal stability of the microbiota over time in established PsA on treatment compared to matched PsC controls.

Objectives: To explore the temporal stability of gut microbiota composition and reveal associations with PsA compared to PSC while on stable on treatment with methotrexate.

Methods: Patients with PsA and PsC were recruited to the study if they had been on a stable dose of methotrexate for 6 months. Bacterial DNA was extracted and the V3-V4 hypervariable region of the 16S rRNA was amplified and sequenced on MiSeq. The resultant data was analysed using a bespoke bioinformatics pipeline and taxa were assigned using the Ribosomal Database Project classifier according to the SILVA119 database. The Wilcoxon rank sum test was used to assess alpha diversity indices, while permanova testing using Bray Curtis distance and DESeq2 values corrected for false-discovery rate (FDR) were used to compare beta diversity indices after removing low abundance $(<0.5 \%)$ Operational Taxonomic Units (OTU). The ALDEx2 analysis package was used to assess effect size.

Results: Stool samples were available 9 PsA $(n=13)$ and 6 PsC $(n=12)$ individuals. Second stool samples were also obtained from the PsA $(n=5)$ and PsC $(n=4)$ groups.

No significant difference in the alpha diversity indices was observed between PsA and PSC. The beta diversity index showed no significant difference between the two conditions using permanova test. However, using the DESeq2-FDR analysis,

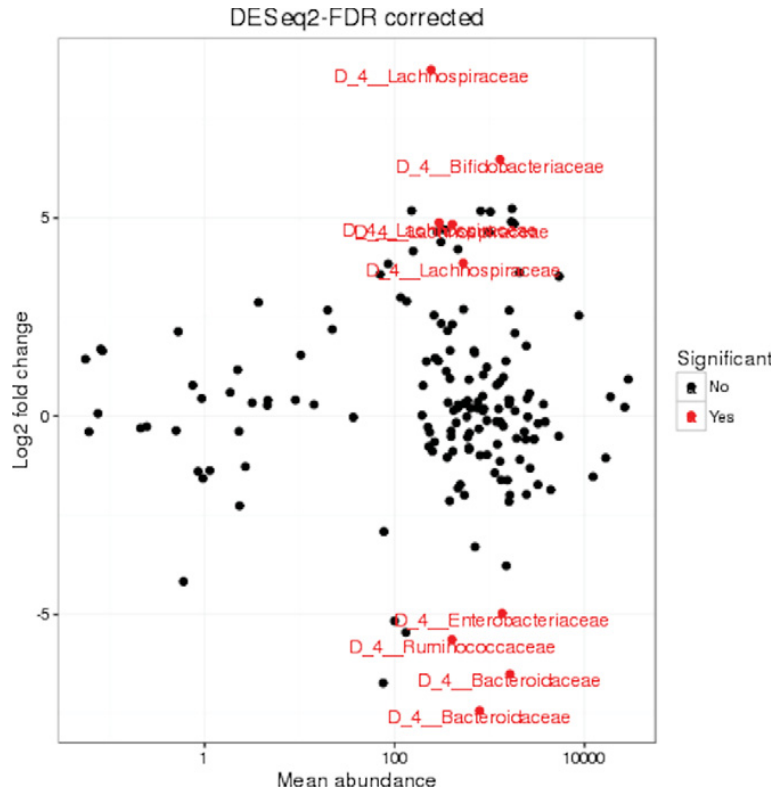

Table 1. Baseline Demographics

\begin{tabular}{lcc}
\hline Demographic variables & PsA $(\mathrm{n}=9)$ & PsC $(\mathrm{n}=6)$ \\
\hline Age mean (range) yrs & $56.8(40-72)$ & $58.5(27-79)$ \\
Gender Female (\%) & $2(22)$ & $4(67)$ \\
Duration of Psoriasis mean (median) yrs & $23.7(26)$ & $26.7(30)$ \\
Type 1 Psorasis (Age at onset <40yrs) (\%) & $7(77.8)$ & $3(50)$ \\
\hline
\end{tabular}

8 OTUs were identified which had significantly $(p<0.01)$ different abundances in PsA compared to PsC. The taxa (Lachnospiraceae \& Ruminococcaeceae) predominantly belonged to the Firmicutes phylum, family Lachnospiraceae and Actinobacteria phylum, family Bifidobacteriaceae (Bifidobacteriaceae). The significant OTUs with DESEq2 had an effect size $>1$ using ALDEx2 but the BH $p$-value was not significant $(p<0.01)$, which may be due to the small sample size. There were no significant differences in the diversity measures over time.

Conclusions: These results suggest that a gut enterotype with predominant Firmicutes/Actinobacteria composition is associated with stable/well controlled disease and is stable over time. This requires replication in a larger cohort.

References:

[1] Lindqvist, 2006.

[2] Scarpa, 2000.

[3] Scher, 2015

Disclosure of Interest: None declared

DOI: 10.1136/annrheumdis-2017-eular.3404

\section{AB0116 IDENTIFYING THE AS PATIENT AT RISK: IS AORTIC ROOT DILATATION ASSOCIATED WITH HLA-B27?}

M. Baniaamam ${ }^{1}$, S. Heslinga ${ }^{2}$, T. Konings ${ }^{3}$, O. Kamp ${ }^{3}$, I. van der Horst-Bruinsma ${ }^{1}$, M. Nurmohamed ${ }^{1} .{ }^{1}$ Rheumatology; ${ }^{2}$ Reade/VU medical centre; ${ }^{3}$ Cardiology, VU Medical Centre, Amsterdam, Netherlands

Background: Cardiac involvement is more common in Ankylosing Spondylitis (AS) patients with HLA-B27 genotype, especially aortic valvular regurgitation (AVR). AVR in AS is caused by aortic root dilatation and fibrotic thickening of the aortic cusps, both linked to inflammation. Inflammation of the aortic root might lead to a weakening in aortic wall strength and dilatation with AVR. Severe AVR can result in heart failure and is an indication for valve replacement or repair. The prevalence of AVR in AS is estimated at $14-18 \%$, which is significantly higher compared to the general population. Therefore, some advocate regular echocardiographic screening of AS patients [1]. However, the cost-benefit of echocardiographic screening in AS is currently unknown and the precise effect of AS specific cardiac pathology on clinically overt cardiovascular morbidity and mortality remains to be elucidated. Hence, we should aim to identify a specific "at risk" AS population that might benefit from routine echocardiographic monitoring. Objectives: Primary: To assess the association between the aortic root diameter in HLA-B27 positive versus HLA-B27 negative patients.

Secondary: To assess the association between the aortic root diameter with disease duration and inflammation biomarkers.

Methods: We performed a cross-sectional study in AS patients between 50-75 years who were recruited from a large rheumatology outpatient clinic. Patients underwent echocardiography, with 2D, spectral and colour flow Doppler. The aortic root was measured at sinuses of Valsalva during diastole. The aortic root diameter was corrected for body surface area (BSA). Correlation between aortic root diameter/BSA and disease duration and inflammation biomarkers were assessed.

Results: 132 Consecutive AS patients were included with a mean age of 60.5 years, of whom $110(83 \%)$ were HLA-B27 positive. The median aortic

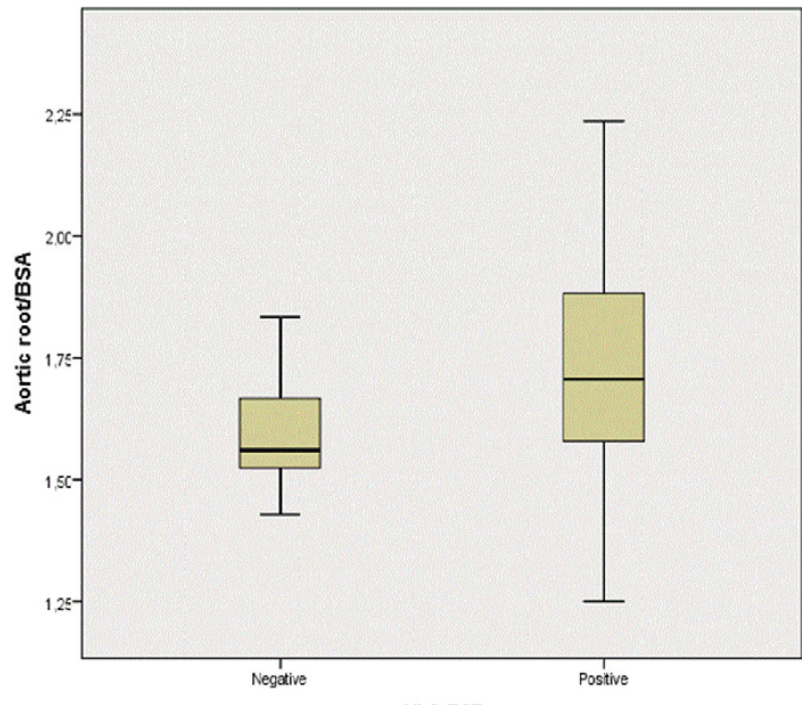

HLA-B27 
root diameter/BSA ratio was significantly higher in HLA-B27 positive patients compared to HLA-B27 negative patients: $1.75 \pm 0.22 \mathrm{~mm}$ vs. $1.61 \pm 0.14 \mathrm{~mm}$ $(p=0.001)$. Eight AS patients $(6 \%)$ had aortic root dilatation $(>2.1 \mathrm{~mm}$ corrected for BSA), who were all HLA-B27 positive. Patients with and without aortic root dilatation did not significantly differ in age or disease duration. The median aortic root diameter/BSA ratio was correlated with disease duration $(r=0.229, p=0.012)$, but not with inflammatory biomarkers.

Conclusions: HLA-B27 positive AS patients with a long disease duration have an increased aortic root diameter and a higher risk for aortic root dilatation compared to HLA-B27 negative AS patients. The risk of developing aortic root dilatation in AS patients is comparable to patients with essential hypertension (6\%)[2].

Future prospective studies should assess which AS patients phenotype are at the highest risk of this AS specific cardiac disease, thus candidates for regular echocardiographic screening.

\section{References:}

[1] Klingberg et al. Aortic Regurgitation Is Common in AS: Time for Routine Echocardiography Evaluation? Am J Med 2015Nov;128(11)1244-1250.

[2] Cuspidi $\mathrm{C}$ et al. Prevalence and correlates of aortic root dilatation in patients with essential hypertension. J Hypertens 2006 Mar;24(3)573-80.

Disclosure of Interest: None declared

DOI: 10.1136/annrheumdis-2017-eular.4616

\section{AB0117 CADHERIN-11 MRNA EXPRESSION IS INCREASED IN THE PERIPHERAL BLOOD OF PATIENTS WITH ACTIVE SPONDYLARTHROPATHY: A PILOT STUDY}

N.I. Vlachogiannis, P.F. Christopoulos, G. Bamias, K. Fragiadaki, M. Tektonidou, P.P. Sfikakis. Joint Academic Rheumatology Program, National and Kapodistrian University of Athens Medical School, Athens, Greece

Background: Cadherin-11 is a key regulator of synovial architecture and has a central role in the formation of the rheumatoid pannus. Immunohistochemical studies have shown upregulation of cadherin-11 in the synovium of patients with seronegative spondylarthropathies (SpA), comparable to that in rheumatoid arthritis (RA), as well as in the intestinal tissue of patients with inflammatory bowel disease (IBD). Moreover, cadherin-11 mRNA transcripts have been identified in the peripheral blood of patients with RA and independently associated, among various disease characteristics, with the presence of active inflammation in multiple joints [1].

Objectives: To test the hypothesis that cadherin-11 mRNA transcripts are increased in the peripheral blood of patients with active SpA and search for possible associations with clinical features.

Methods: Fifteen patients with active SpA (BASDAl>4), aged between 21 and 71 years, 11 men, and 30 age- and gender-matched healthy controls were examined. Peripheral whole blood samples $(3 \mathrm{ml})$ were subjected to CDNA synthesis and cadherin-11 mRNA expression was quantified by real-time PCR. Available cDNA from 33 IBD patients without axial or peripheral active arthritis served as disease control and were studied in parallel.

Results: Cadherin-11 mRNA was detected in the peripheral blood of 9/15 $(60 \%)$ patients with SpA versus 5/30 (17\%) healthy controls and 10/33 (30\%) patients with IBD (SpA vs healthy controls $p: 0.006$, SpA vs IBD p: 0.06). Notably, cadherin-11 was not associated with BASDAI or skin/nail psoriasis present in 10 patients, but was detected with increased frequency among SpA patients with clinically active peripheral arthritis at the time of sampling (7 out of 10,70\%) than the remaining patients (2 out of $5,40 \%$ ). Moreover, cadherin-11 positivity associated significantly with increased erythrocyte sedimentation rate in SpA but not in IBD. Conclusions: Cadherin-11 mRNA is upregulated in the peripheral blood of patients with SpA and may correlate with "spreading" of inflammation in peripheral joints. Since an anti-cadherin-11 mAb is in early clinical development for RA, further studies in patients with inflammatory arthritis are warranted.

\section{References:}

[1] Sfikakis PP, Christopoulos PF, Vaiopoulos AG, et al. Cadherin-11 mRNA transcripts are frequently found in rheumatoid arthritis peripheral blood and correlate with established polyarthritis. Clin Immunol 2014;155:33-41. doi:10.1016/j.clim.2014.08.008.

Disclosure of Interest: N. Vlachogiannis: None declared, P. Christopoulos: None declared, G. Bamias: None declared, K. Fragiadaki: None declared, M. Tektonidou: None declared, P. Sfikakis Grant/research support from: Educational grant from Janssen

DOI: 10.1136/annrheumdis-2017-eular.3650

\section{AB0118 THE LYMPHATIC SYSTEM: A GATEKEEPER FOR MIGRATION OF PATHOGENIC T-CELLS TOWARDS SYNOVIAL JOINTS AND ENTHESES IN PSORIASIS}

R. Bisoendial ${ }^{1}$, E. Prens ${ }^{2}$, A. Mus ${ }^{3}$, P. Asmawidjaja ${ }^{3}$, N. Davelaar ${ }^{3}$ A. Hofman ${ }^{4}$, J.-B. Jaquet ${ }^{4}$, J. Hazes ${ }^{3}$, M. Kok ${ }^{5}$, E. Lubberts ${ }^{3} .{ }^{1}$ Department of Rheumatology, Maasstad hospital and Erasmus Medical Center; ${ }^{2}$ Department of Dermatology; ${ }^{3}$ Department of Rheumatology, Erasmus University Medical Center; ${ }^{4}$ Department of Plastic surgery; ${ }^{5}$ Department of Rheumatology, Maasstad hospital, Rotterdam, Netherlands

Background: The factors underlying the transition of psoriasis ( $\mathrm{PsO}$ ) to psoriatic arthritis (PsA) are poorly understood. The lymphatic system may control the homing of disease-associated T-cells to skin and extra-cutaneous sites like synovial joints and entheses.

Objectives: To study the capacity of lymphatic endothelial cells (LEC) to regulate T-cell homing capabilities in PsA.

Methods: Human dermal LEC $\left(0.5 \times 10^{4}\right)$, and fibroblast-like synoviocytes of a

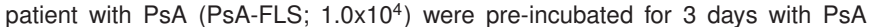
synovial fluid (PsA-SF; 0/10/20\% v/v) and co-cultured with $2.5 \times 10^{4}$ allogeneic $\mathrm{CD} 4+\mathrm{CD} 45 \mathrm{RO}+\mathrm{CD} 25-\mathrm{T}$-cells that were sorted from peripheral blood mononuclear cells of 3 donors (with or without stimulation with $\alpha \mathrm{CD} 3 / \alpha C D 28$ ). After $72 \mathrm{~h}$, T-cells were analyzed by flow cytometry. The CCR6+ T-helper (Th) subsets Th17.1 (CCR4-/CXCR3+), Th17/Th22 (CCR4+/CXCR3-), Th17 (CCR4+/CXCR3/CCR10-) and Th22 (CCR4+/CXCR3-/CCR10+), and CCR6- subsets Th1 (CCR4/CXCR3+), and Th2 (CCR4+/CXCR3-) were identified. We also looked at cutaneous lymphocyte- associated antigen (CLA). IL-17A, IL-22, and TNF protein levels were determined by ELISA. Statistical analysis included unpaired t-test (two-sided) for two-group comparison or one-way ANOVA with the Tukey-Kramer post hoc test for multi-group comparisons.

Results: Stimulation of CD4+CD45RO+ T-cells in co-culture with PsA-FLS skewed towards the CCR6+ subset Th17/Th22, which were predominantly Th17 cells. Th17 differentiation upon stimulation was suppressed in co-culture with LEC, even when LEC were pre-incubated with PsA-SF. T-cell stimulation in co-culture with LEC, as compared to PsA-FLS, promoted the generation of the Th22 subset. Upon co-culture with untreated LEC, stimulated T-cells showed higher expression of the skin homing receptor CLA than those that were co-cultured with PsA-FLS. The proportional reduction in CLA expression on T-cells in the co-cultures with LEC pre-incubated with PSA-SF $20 \%$ was comparable to PsA-FLS, however LEC conserved CLA expression on CD4+CD45RO T-cells at a higher level than PsA-FLS; this phenomenon particularly affected the CCR6+ T-cells. In line, a trend towards lower IL-17A and higher IL-22 levels were observed in the co-cultures with LEC that were pretreated with PSA-SF 20\%, as compared to the co-culture with PsA-FLS. No differences were seen for TNF protein levels.

Conclusions: LECs are directly involved in T-cell differentiation and homing capabilities, as shown by suppression of Th17 differentiation upon stimulation, as compared to PsA-FLS. Also, LEC promoted Th22 generation, and conserved CLA expression in CCR6+ T-cells, even when LEC were preincubated with PSA-SF. Studies are underway to confirm that LECs from relevant biological tissues (e.g. synovium and lymph nodes) are critical for tissue-restricted T-cell migration to skin and synovial membranes in PsA.

Disclosure of Interest: None declared

DOI: 10.1136/annrheumdis-2017-eular.5360

\section{AB0119 ANTI-FIBROTICS TO PREVENT NEW BONE FORMATION IN SPONDYLOARTHRITIS: PROOF OF CONCEPT USING PIRFENIDONE IN CELL CULTURE MODELS}

J. Laustsen ${ }^{1,2}$, S. Lomholt ${ }^{3}$, P. Andersen ${ }^{3}$, J. Kelsen ${ }^{4}$, T.W. Kragstrup ${ }^{1}$. ${ }^{1}$ Department of Rheumatology, Aarhus University Hospital; ${ }^{2}$ Department of Biomedicine; ${ }^{3}$ Aarhus University; ${ }^{4}$ Aarhus University Hospital, Aarhus $C$, Denmark

Background: The pathogenesis of spondyloarthritis $(\mathrm{SpA})$ involves both inflammation and new bone formation in the spine. In line with this, the disease has been characterized as both inflammatory and fibrotic. Current treatment including inhibitors of tumour necrosis factor alpha (TNF $\alpha$ ) seem to dampen inflammation while new bone formation can progress. Therefore, there is an unmet therapeutic need for the treatment of new bone formation in SpA. Fibrosis is mediated by myofibroblasts and new bone formation is the result of increased osteoblast mineralization and decreased osteoclast bone degradation. Here, we evaluate the potential effect of the newly approved anti-fibrotic agent pirfenidone (Esbriet, Pirespa) on fibrosis and new bone formation in cell culture models of SpA.

Objectives: We hypothesized that pirfenidone inhibits SpA myofibroblast formation and activity and osteoblast mineralization.

Methods: Synovial fluid mononuclear cells from patients with $\operatorname{SpA}(n=6)$ were included for culturing fibroblast-like synovial cells (FLSs) while osteoblasts were purchased. The cells were cultured with pirfenidone in increasing concentrations $(0.25,0.5$, and $1.0 \mathrm{mg} / \mathrm{ml})$ with or without stimulation with tumor necrosis factor alpha (TNF $\alpha$ ), transforming growth factor beta $(\mathrm{TGF} \beta)$, or interferon gamma $(\mathrm{INF} \gamma)$. The proliferation of FLSs was analyzed with light microscopy and flow cytometry using the marker Ki67. The differentiation and activation of FLSs was assessed with flow cytometry, a proteome profiler assay and enzyme-linked immunosorbant assays. The mineralization capacity of the osteoblasts was measured as deposition of hydroxyapatite.

Results: Pirfenidone reduced the Ki67 expression 7.1-fold in untreated FLSs $(\mathrm{p}=0.001)$ and 11.0 -fold in FLSs stimulated with TGF $\beta$, TNF $\alpha$, and INF $\gamma(\mathrm{p}=0.022)$. PFD further inhibited TGF $\beta$ induced upregulation of $\alpha$ SMA (Figure 2A) and INF $\gamma$ induced upregulation of HLA-DR (Figure $2 \mathrm{C}$ ) in all cultures. There was no difference between the percentage of ICAM-1 positive FLSs in cultures treated with or without pirfenidone. In supernatants from FLSs stimulated with TGF $\beta$, TNF $\alpha$, and INF $\gamma$ a total of 12 cytokines or chemokines had values above the detection limit in the membrane-based antibody array. Pirfenidone decreased the secretion of 3 of these 12 cytokines or chemokines more than 2-fold. The changes in secretion of monocyte chemoattractant protein 1 (MCP-1) and chitinase-3-like 